\title{
Neonatal Sepsis and Meningitis Caused by Elizabethkingia
}

\author{
Chanchal Kumar $^{1} \cdot$ Apurba Sankar Sastry $^{2} \cdot$ Nishad Plakkal $^{1}$ (D) \\ Received: 3 November 2020 / Accepted: 10 March 2021 / Published online: 7 April 2021 \\ (C) Dr. K C Chaudhuri Foundation 2021
}

To the Editor: Elizabethkingia species are saprophytic gramnegative rods ubiquitous in soil and water but can occasionally cause sepsis and meningitis in infants [1]. These bacteria can colonize water sinks and respirators, and contaminate saline solutions, lipid solutions, and reconstituted antibiotics [2, 3]. Clinical reports from neonatal intensive care units (NICUs) are few [4]. In two outbreaks in our NICU, we noticed that this bacterium frequently infected neonates without the usual risk factors for nosocomial infection like intravenous catheters or ventilator support and had a high propensity to cause meningitis. The number of affected neonates were 2 and 30 in the two outbreaks, respectively. A total of 47 isolates of Elizabethkingia species were identified from the blood and cerebrospinal fluid (CSF) of the 32 neonates (36 blood and 11 nonaugmented CSF cultures). Nine infants had growth in both blood and CSF. We noted meningitis, requirement of ventriculo-peritoneal shunt placement, and mortality in 19 , 4 , and 6 infants, respectively. Isolation of the organism was done by using BacT/ALERT, and VITEK-2 system was used for species identification and for antibiotic sensitivity pattern. The mean time to positivity for growth from blood was 12.9 (range 2-23) h. E. anophelis was isolated in half the cases and E. meningoseptica in the rest. Susceptibility to amikacin, ciprofloxacin, cefoperazone-sulbactam, and piperacillintazobactam was noted in $68 \%, 72 \%, 72 \%$, and $30 \%$, respectively. We noted universal resistance to carbapenems (intrinsic resistance) and universal sensitivity to levofloxacin and minocycline. Line-listing of the cases helped to narrow down the source in the first outbreak. Extensive environmental microbiological investigations resulted in isolation of E. meningoseptica from one of the breast pumps. Cleaning

Nishad Plakkal

plakkal@gmail.com

1 Department of Neonatology, Jawaharlal Institute of Postgraduate Medical Education \& Research, Puducherry 605006, India

2 Department of Microbiology, Jawaharlal Institute of Postgraduate Medical Education \& Research, Puducherry, India protocols for reusable accessories were changed and reinforced, including cleaning of the tubing and container and capping of the tubing after use. However, the source of infection could not be identified in the second outbreak, despite repeated extensive environmental testing.

\section{Declarations}

Ethical Approval Outbreak investigation is an integral part of clinical management; hence ethics approval was not obtained. No personal identifiers have been retained. All procedures performed in the study were in accordance with the ethical standards of the institutional ethics committee and with the 1964 Helsinki declaration and its later amendments.

Conflict of Interest None.

\section{References}

1. Issack MI, Neetoo Y. An outbreak of Elizabethkingia meningoseptica neonatal meningitis in Mauritius. J Infect Dev Ctries. 2011;5:834-9.

2. Ceyhan M, Celik M. Elizabethkingia meningosepticum (Chryseobacterium meningosepticum) infections in children. Int $\mathbf{J}$ Pediatr. 2011;2011:215237.

3. Tak V, Mathur P, Varghese P, Misra MC. Elizabethkingia meningoseptica: an emerging pathogen causing meningitis in a hospitalized adult trauma patient. Indian J Med Microbiol. 2013;31: 293-5.

4. Shailaja VV, Reddy AK, Alimelu M, Sadanand LNR. Neonatal meningitis by multidrug resistant Elizabethkingia meningosepticum identified by 16 s ribosomal RNA gene sequencing. Int J Pediatr. 2014;2014:918907.

Publisher's Note Springer Nature remains neutral with regard to jurisdictional claims in published maps and institutional affiliations. 